\title{
GOBERNANZA Y DESARROLLO INCLUSIVO EN MATERIA DE ACCESIBILIDAD Y REHABILITACIÓN DE CIUDADES EMERGENTES
}

\author{
Nieves Navarro Cano \\ Profesora Titular Universidad \\ Directora Catedra Empresa Accesibilidad en la Edificación \\ Universidad Politécnica Madrid. UPM
}

Artículo Recibido: 25/11/2016

Artículo Aceptado: 28/12/2016

\section{Resumen}

Al tratar de innovación urbana de las ciudades, se incorpora en el concepto de ciudades inteligentes, a aquellas que usan la tecnología para incrementar la eficiencia y la sostenibilidad de sus servicios, sin embargo, en pocas ocasiones se integra la valoración del criterio de ciudad "inclusiva y accesible".

El objetivo de éste artículo es, trasladar la necesidad de incluir éste nuevo concepto, en la "gobernanza de las ciudades", un aspecto esencial, que garantiza la inclusión de todas las personas, cualquiera que sea su condición, lo cual implica adherir los términos de accesibilidad y la diversidad. Se pretende contemplar que los derechos escritos, reconocidos para las personas con discapacidad, se realicen en la realidad para conseguir la participación activa de todos en la ciudad, sea cual sean sus condiciones y capacidades.

Se resalta la necesidad de hablar de inclusión de personas con otras capacidades o diversidad funcional, como concepto innovador que no discrimina a nadie e incluye a todos, es decir como elemento dinamizador de aplicación a la gobernanza de la ciudad.

WPS RI-SHUR, n4, 2016, vol.1, ISSN: 2387-1768 


\title{
WPSReview International on Sustainable Housing and Urban Renewal
}

(RI-SHUR)

Se pretende incorporar un nuevo concepto en el diseño de la gobernanza de las ciudades, que no es otro que la ciudad destinada para los ciudadanos, como elemento esencial que rige su misión y valor, en el que es preciso incorporar a todos los ciudadanos, sin exclusión social, por lo que debemos garantizar la inclusión de las personas con diversidad funcional.

\begin{abstract}
When dealing with urban innovation in cities, it is incorporated into the concept of smart cities, those that use technology to increase the efficiency and sustainability of its services, however, on a few occasions integrates the assessment of the criterion of city " Inclusive and accessible ".
\end{abstract}

The purpose of this article is to translate the need to include this new concept in the "governance of cities", an essential aspect, which guarantees the inclusion of all people, whatever their condition, which implies adhering to the terms Accessibility and diversity. It is intended to contemplate that written rights, recognized for people with disabilities, be realized in reality to achieve the active participation of all in the city, whatever their conditions and capabilities.

The need to speak of the inclusion of people with other capacities or functional diversity is highlighted as an innovative concept that does not discriminate against anyone and includes all, that is to say as a dynamic element of application to the city's governance.

It is intended to incorporate a new concept in the design of city governance, which is none other than the city destined for citizens, as an essential element that governs its mission and value, in which it is necessary to incorporate all citizens, without Exclusion, so we must ensure the inclusion of people with functional.

\section{Palabras Clave}

Gobernanza, Ciudades, Inclusión, Accesibilidad, Ciudades Inteligentes, sociedad, discapacidad.

\section{Key Words}

Governance, Cities, Inclusion, Accessibility, Intelligent Cities, society, disability

WPS RI-SHUR, no4, 2016, vol.1, ISSN: 2387-1768 


\section{WPSReview International on Sustainable Housing and Urban Renewal}

(RI-SHUR)

\section{1.- Introducción. Marco Teórico}

El escenario demográfico actual muestra la realidad actual para los próximos cincuenta años, basado en dos factores sumamente relacionados: el incremento de la población en las ciudades y su concentración en las áreas urbanas. Por otra parte, en países en desarrollo se constata una migración masiva de las zonas rurales a las urbanas.

Entre 1950 y 2011, la población urbana aumentó de forma considerable, casi cinco veces. Según datos de Naciones Unidas, en el 2015 el 75\% de la población mundial vivía en las ciudades. En Europa se puede llegar a alcanzar el $80 \%$ en 2020 . Por otra parte, en países en desarrollo una migración masiva de las zonas rurales a las urbanas. Actualmente más del $5 \%$ de las personas viven en ciudades, se espera que en 2050 esta proporción aumente a un $70 \%$.

Según Johannes Hahn, Comisario Europeo de Política Regional más de dos tercios de la población europea vive en zonas urbanas. Las ciudades son lugares donde surgen problemas y se encuentran soluciones. Son un terreno fértil para la ciencia y la tecnología, para la cultura y la innovación, para la creatividad individual y colectiva, y también para mitigar el impacto del cambio climático. Sin embargo, las ciudades también son lugares donde se concentran problemas como el desempleo, la segregación y la pobreza.

El concepto de la nueva ciudad o ciudad inteligente surge en respuesta a la necesidad de que las ciudades evolucionen hacia sistemas cada vez más sostenibles y eficientes a través del uso de las tecnologías de la información y de la comunicación. Las diversas interpretaciones del concepto de "Ciudades Inteligentes", se destaca la interpretación de Giffinger en 2007, asumida por el propio Parlamento Europeo en 2014, en su estudio de Mapping Smart Cities in the European Union, que considera que la "ciudad inteligente" se define como una ciudad con un buen desempeño en la construcción de una visión de futuro para la economía, la gente, el gobierno, la movilidad, el medioambiente y la vida de la ciudad en general.

El término de gobernanza urbana, del inglés, urban governance, se debe analizar las estructuras actuales implantadas entre los actores de la ciudad; considerando entre estos actores al gobierno local, a los dirigentes políticos, las empresas, los dirigentes sociales, los académicos, los colegios profesionales y demás expresiones de la sociedad civil organizada.

WPS RI-SHUR, n4, 2016, vol.1, ISSN: 2387-1768

NÚMERO MONOGRÁFICO SOBRE GOBERNANZA URBANA/SPECIAL ISSUE ON GOVERNANCE 


\section{WPSReview International on Sustainable Housing and Urban Renewal}

(RI-SHUR)

El proceso de reflexión de la gobernanza de Ciudades de futuro debe servir de inspiración para los responsables de la elaboración de políticas y para los profesionales involucrados en el desarrollo urbano, ya sea a nivel local, regional, nacional o europeo. La ciudad Inclusiva exige la participación ciudadana como objetivos esenciales, que implica la inclusión de todos, sea cual sea su condición, lo que obliga a incluir a la diversidad.

Las orientaciones generales que lideran la estructura urbana y su desarrollo, se caracteriza por tres factores esenciales: la ciudad económica, que afectan factores de competitividad, ciudades para el ciudadano, que considera el capital social y humano y la ciudad de la movilidad, que incorpora el factor del transporte. Hay otros factores que se deben señalar la "smart living" calidad de vida; y "smart governance", participación (toma de decisiones; servicios públicos y sociales; transparencia y estrategias políticas).

Si analizamos los factores que intervienen en el diseño de las ciudades inteligentes sostenibles, comprobamos que, desde su inicio, se basa en aspectos relevantes de mejoras que garantizan una facilidad de participación del ciudadano, progreso del transporte y avances en las tecnologías de la información (TICS), sin embargo las estrategias planificadas se olvidan de incluir a una parte importante de usuarios de la ciudad, las personas con discapacidad.

Las ciudades necesitan adoptar enfoques de planificación y desarrollo que integren las dimensiones sociales, ambientales, económicas y territoriales del desarrollo urbano. La competitividad de la ciudad debe ser inclusiva, sin excluir a ninguno de los grupos más vulnerables, teniendo en cuenta a las personas desde la diversidad y admitiendo las capacidades de los distintos colectivos.

Las recomendaciones de la Agenda Territorial 2020 sobre la inclusión del desarrollo urbano en un contexto territorial ligado a la Estrategia Europa 2020, y sobre el fomento del desarrollo territorial equilibrado y el uso de planteamientos de desarrollo integrado en ciudades y regiones rurales y específicas, debe adoptar el desafío, no solo del desarrollo territorial, debe admitir la gobernanza a varios niveles, incluyendo tanto la gestión de recursos energéticos, expansión urbana, aplicación de las tecnologías de la información y mejoras de sus servicios, incorporando a todos los ciudadanos. Las ciudades deben adoptar la gobernanza de forma gradual socialmente innovadora, integrada, accesible e inclusiva, debiendo trabajar de forma conjunta adoptando normativas y políticas que reconozcan los derechos de todos.

WPS RI-SHUR, nㅇ4, 2016, vol.1, ISSN: 2387-1768

NÚMERO MONOGRÁFICO SOBRE GOBERNANZA URBANA/SPECIAL ISSUE ON GOVERNANCE 


\section{WPSReview International on Sustainable Housing and Urban Renewal}

(RI-SHUR)

\section{2.- Metodología}

La metodología de análisis aplicada para la recopilación de la información incorporada, se basa, principalmente, de la evidencia de falta de inclusión de las personas con discapacidad en la gobernanza de las ciudades, constatados en los datos recopilados que afectan a distintas ciudades, con el objetivo de localizar iniciativas de carácter de "Ciudades Inteligentes", que sean fiel reflejo de la realidad y del nivel del desarrollo de cada una de las ciudades.

El análisis realizado parte de la realización de un estudio desarrollado en distintas ciudades, organizado por datos recogidos de distintas ciudades que han generado una muestra en el estudio realizado.

\section{3.- Desarrollo Trabajo}

Se define la Ciudad Inteligente como aquella ciudad que utiliza los avances tecnológicos para mejorar la calidad de vida de los ciudadanos. La Ciudad Inteligente se ha convertido en un objetivo de los ayuntamientos, se considera el nuevo paradigma de modernización y progreso económico.

Si La Ciudad Inteligente se basa en la mejora de vida del ciudadano y en la participación activa del ciudadano, debemos incluir a Todos los ciudadanos, sin excluir a ningún colectivo. En consecuencia, hay que garantizar la inclusión de las personas con discapacidad y mayores

Uno de los mayores retos según declaró en 2014 Michael Sorkin en la Jornadas de "Iniciativa de Ciudades Emergentes y Sostenibles", organizadas por el Banco Interamericano de Desarrollo. es la aparición de megaciudades y la expansión de la población, agregando un millón de personas cada semana a nuestras ciudades, sin disponer de soluciones adecuadas para alojamiento y disponibilidad de empleo.

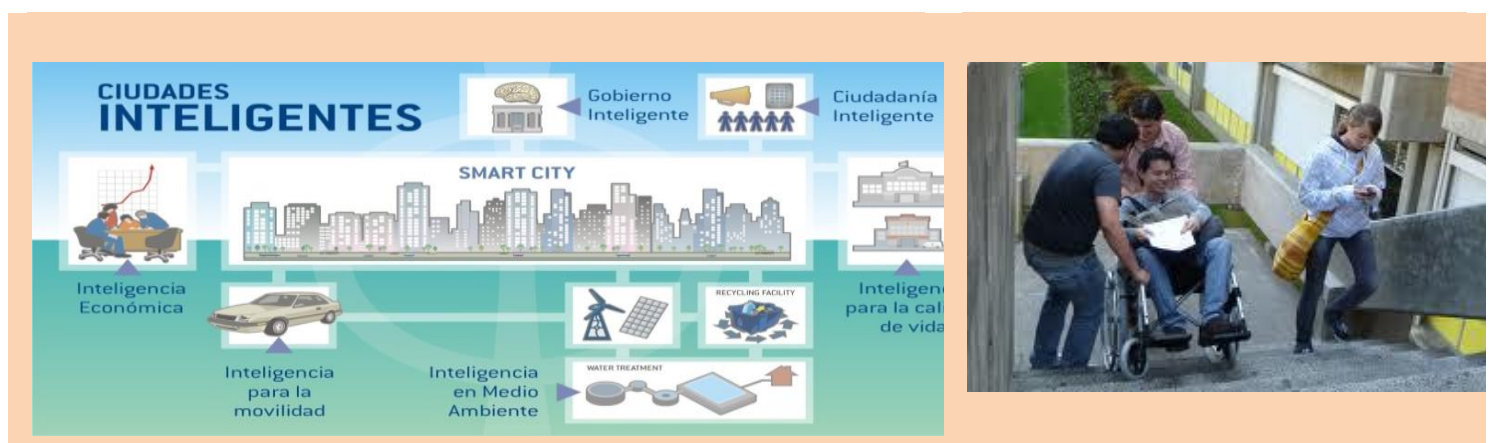

WPS RI-SHUR, n4ㄴ, 2016, vol.1, ISSN: 2387-1768

NÚMERO MONOGRÁFICO SOBRE GOBERNANZA URBANA/SPECIAL ISSUE ON GOVERNANCE 


\section{WPSReview International on Sustainable Housing and Urban Renewal}

(RI-SHUR)

A esta situación hay que añadir la necesidad de buscar soluciones para que el desarrollo urbano sea accesible e inclusivo para las mil millones de personas del mundo que tienen discapacidad, la mayor parte de los cuales viven en ciudades, garantizando una CIUDAD INTEGRAL INCLUSIVA, ACCESIBLE Y AMIGABLE.

\section{1.- Datos sobre Discapacidad.}

La Clasificación Internacional del Funcionamiento de la Discapacidad y de la Salud (CIF), aprobada en 2001, adoptada como marco conceptual para el Informe de la OMS, define la discapacidad como un término genérico que engloba deficiencias, limitaciones de actividad y restricciones para la participación.

La discapacidad señala los aspectos negativos de la interacción entre personas con un problema de salud (como parálisis cerebral, síndrome de Down o depresión) y factores personales y ambientales (como actitudes negativas, transporte y edificios públicos inaccesibles, y falta de apoyo social).

Esta nueva definición supone un gran avance en el ámbito de la accesibilidad y la discapacidad, al relacionar la discapacidad con el entorno ambiental. Es decir, se excluye la discapacidad, como término que considera las deficiencias de las personas como un factor que impide la utilización de la ciudad, e incorpora un nuevo concepto, al introducir que la discapacidad surge debido a la falta de adaptación medioambiental, de los entornos, productos y servicios y, recalca que la falta de adaptación de la ciudad es la que ocasiona que personas con otras capacidades, puedan utilizar espacios determinados.

El cambio de criterios y valores es primordial para entender cómo actuar, ya que considera la necesidad de adaptación de los espacios, entornos, elementos, etc. como una responsabilidad social, en la gobernanza de las ciudades de forma que permita un uso para todos, conforme recoge la normativa, responsabilizando a la falta de adaptación, como factor esencial, quién imposibilita su utilización

Hay aproximadamente mil millones de personas con discapacidad, lo que supone el 15\% Casi 200 millones a nivel mundial tienen dificultades importantes. En España hay un total de 3.528.221 personas con alguna discapacidad, lo que supone una global de prevalencia de un 9\%. En España en el año 2050 según la OMS el colectivo de personas con más de 65 años, se elevará hasta los 16 millones.

Según estadísticas en 2052 en España el grupo de personas mayores de 64 años aumentarán en 7,2 millones de personas. Supone un $89 \%$ y ocupa un $37 \%$ de la población española.

WPS RI-SHUR, n4, 2016, vol.1, ISSN: 2387-1768

NÚMERO MONOGRÁFICO SOBRE GOBERNANZA URBANA/SPECIAL ISSUE ON GOVERNANCE 


\section{WPSReview International on Sustainable Housing and Urban Renewal}

(RI-SHUR)

En el futuro, la discapacidad será un motivo de preocupación, pues su prevalencia aumenta, debido al envejecimiento de la población y supone un aumento mundial de enfermedades (cardiovasculares, diabetes, cáncer, trastornos salud mental, etc...

Según datos de la Organización Mundial de la Salud (OMS)de 2006, para el año 2050 la proporción de personas mayores de 60 años se duplicará, pasará del 11 al $22 \%$ del total; en 2006 la población mayor de 60 años era del 11\% de la población total. En 2050, por primera vez en la historia de la humanidad, habrá más personas mayores que niños en la población.

Los países en desarrollo envejecen a un ritmo mucho mayor que los países desarrollados. Transcurridas cinco décadas, poco más del $80 \%$ de las personas mayores del mundo, vivirán en países en desarrollo, frente al 60\% del año 2005

Esto obliga a aplicar o al menos a considerar que, en el futuro sea necesario crear políticas y programas innovadores, en la "Gobernanza de las ciudades", que garanticen la plena inclusión de las personas con discapacidad y personas mayores de forma activa en la ciudad.

\section{2.-Aplicación de la Ciudad Inclusiva y Accesible}

Una Ciudad innovadora es aquella que pone la ciudad al uso del ciudadano para mejorar su calidad de vida. Es aquella ciudad que utiliza la tecnología para mejorar el bienestar de la comunidad, aplica criterios de sostenibilidad para disponer de edificios autosuficientes que mejoren costes.

Se trata de una ciudad comprometida con su entorno urbano, que aplica tecnologías avanzadas y en la que interviene soluciones tecnológicas innovadoras a disposición de los ciudadanos, considerando a Todos los ciudadanos, sin exclusión de ninguno de ellos.

A todos estos aspectos hay que añadir, para incluir en la gobernanza de las ciudades el factor del uso para todos, la variable de Accesibilidad, para garantizar Ciudades que permiten su uso por Todos los ciudadanos, comprometidas con el cumplimiento de los derechos reconocidos de las personas con discapacidad.

Incorporar a las personas con discapacidad funcional o cognitiva. Es preciso apostar por Ciudades Inteligentes, Inclusivas y Accesibles, creadas para la Diversidad.

WPS RI-SHUR, nㅇ4, 2016, vol.1, ISSN: 2387-1768

NÚMERO MONOGRÁFICO SOBRE GOBERNANZA URBANA/SPECIAL ISSUE ON GOVERNANCE 


\section{WPSReview International on Sustainable Housing and Urban Renewal}

(RI-SHUR)

Una Ciudad inteligente es aquella que pone la ciudad al uso del ciudadano para mejorar su calidad de vida. Es aquella ciudad que utiliza la tecnología para mejorar el bienestar de la comunidad, aplica criterios de sostenibilidad para disponer de edificios autosuficientes que mejoren costes.

Se trata de una ciudad comprometida con su entorno urbano, que aplica tecnologías avanzadas y en la que interviene soluciones tecnológicas innovadoras a disposición de los ciudadanos, de Todos los ciudadanos.

Si definimos como debe ser la gobernanza de una Ciudad Inclusiva y Accesible, podemos decir que es aquella en la que se garantizan y consideran los derechos de las personas con discapacidad y personas mayores. Es aquella que aplica la Accesibilidad Universal y Diseño para Todos en todos sus proyectos, la usabilidad de todos sus productos y servicios y diseña políticas de inclusión social para todos. Es aquella que no hay discriminación, ni barreras físicas y sus diseños permiten su uso a Todas las personas, con seguridad, dignidad y autonomía. de Igualdad en la usabilidad de la ciudad, la empleabilidad, la educación, la sanidad y el uso de las tecnologías de información a disposición del ciudadano.

\section{3.- Claves para una Ciudad Inclusiva y Accesible}

La ciudad Inclusiva y Accesible es aquella en la que se garantizan y consideran los derechos de las personas con discapacidad y personas mayores y aplica la Accesibilidad Universal y Diseño para Todos en todos sus proyectos, la usabilidad de todos sus productos y servicios y diseña políticas de inclusión social para todos.

Es aquella que no hay discriminación, ni barreras físicas y sus diseños permiten su uso a Todas las personas, con seguridad, dignidad y autonomía. Es aquella que respeta los criterios de Igualdad en la usabilidad de la ciudad, la empleabilidad, la educación, la sanidad y el uso de las tecnologías de información a disposición del ciudadano.

Si los pilares de la Gobernanza de una ciudad innovadora e inteligente son la tecnología, los servicios y los ciudadanos, las claves para la gobernanza de una ciudad inclusiva y accesible son la igualdad, la flexibilidad, la accesibilidad y aplicación de "Ajustes Razonables" para la adaptación de una ciudad para todos.

La Igualdad nos garantiza la equidad de derechos y deberes para todos, sin sin discriminación de ningún colectivo. Debe proporcionar igualdad de condiciones para Todos para acceder a los servicios sociales, educación, sanidad y empleabilidad.

WPS RI-SHUR, nㅇ4, 2016, vol.1, ISSN: 2387-1768

NÚMERO MONOGRÁFICO SOBRE GOBERNANZA URBANA/SPECIAL ISSUE ON GOVERNANCE 


\section{WPSReview International on Sustainable Housing and Urban Renewal}

(RI-SHUR)

La Flexibilidad y el disponer de sistemas flexibles de adaptación de las ciudades, permite que personas con algún tipo de discapacidad pueda utilizar productos, entornos y edificios diseñados para todos.

La aplicación de Ajustes Razonables para la adaptación de entornos y espacios, permite la adecuación escalable y personalizada de los espacios y viviendas para fomentar un envejecimiento activo y la inclusión de todos los ciudadanos, considerando la diversidad humana.

El concepto de accesibilidad como herramienta esencial en la aplicación de adaptación de las ciudades, se define como el grado en el que todas las personas pueden utilizar un objeto, un espacio, visitar un lugar o acceder a un servicio, independientemente de sus capacidades físicas o cognitivas. La Accesibilidad está relacionada con la inclusión y el respeto a los derechos de los ciudadanos.

El concepto de Diseño para Todos o Diseño Universal, centra toda su actividad en garantizar espacios, entornos y servicios para Todos, sea cual sea la edad, el género, las capacidades físicas, psíquicas y sensoriales de las personas, garantizando su igualdad de uso por Todos.

El objetivo en ambos casos es la búsqueda de soluciones de diseño para todas las personas, que permitan la inclusión de todos. Comprobamos que la "Accesibilidad Universal y el Diseño para Todos mejora la calidad de vida de todas las personas, con o sin discapacidad, por lo que es un reto conseguir Ciudades Inclusivas y Accesibles para todos, que permitirán compartir espacios de uso común para todos, compartiendo la igualdad y la inclusión de todas las personas, garantizado la presencia de la diversidad funcional.

3.4.-Marco Normativo regulador de la presencia de la Accesibilidad Universal y Diseño para Todos en la Gobernanza de las ciudades

En el ámbito internacional la Convención Internacional sobre los derechos de las personas con discapacidad de Naciones Unidas, obliga a la adaptación de las ciudades para que puedan ser utilizadas por todos. La Convención Internacional sobre los derechos de las personas con discapacidad, fue aprobada el 13 diciembre de 2006 por la Asamblea General de las Naciones Unidas (ONU) y ratificada por España el 3 de diciembre de 2007, entrando en vigor el 3 de mayo de 2008. La Convención supone la ratificación del derecho de las personas con discapacidad y la obligación de España en su aplicación.

WPS RI-SHUR, n4, 2016, vol.1, ISSN: 2387-1768

NÚMERO MONOGRÁFICO SOBRE GOBERNANZA URBANA/SPECIAL ISSUE ON GOVERNANCE 
En el ámbito nacional debemos destacar las que a continuación se detallan:

El Real Decreto 505/2007, de 20 de abril define las Condiciones Básicas de Accesibilidad y no Discriminación de las Personas con Discapacidad para el Acceso y Utilización de los Espacios Públicos Urbanizados y Edificaciones, resaltamos la Disposición Final Quinta que dice "Las condiciones básicas de accesibilidad y no discriminación para el acceso y utilización de los espacios públicos urbanizados y edificaciones que se aprueban en virtud del presente real decreto serán obligatorias a partir del día 1 de enero de 2010 para los espacios públicos urbanizados nuevos y para los edificios nuevos, así como para las obras de ampliación, modificación, reforma o rehabilitación que se realicen en los edificios existentes, y a partir del día 1 de enero de 2019 para todos aquellos espacios públicos urbanizados y edificios existentes que sean susceptibles de ajustes razonables ...."

El Real Decreto Legislativo 1/2013 de 29 noviembre por el que se aprueba el Texto Refundido de la Ley General de Derechos de las personas con Discapacidad y de su Inclusión Social - LGD. Establece la obligatoriedad de adecuar las condiciones básicas de Accesibilidad en los edificios existentes, en lo que sea susceptible de Ajustes Razonables (Art. 23). En los plazos y términos que se establezca reglamentariamente (Art. 25) y, como máximo, antes del 4 de diciembre de 2017.

Añade tendrán carácter obligatorio...vengan impuestas por las Administraciones Públicas o solicitadas a instancia de los propietarios, las siguientes actuaciones:"

"b) Las obras y actuaciones que resulten necesarias para garantizar los ajustes razonables en materia de accesibilidad universal $y$, en todo caso, las requeridas a instancia de los propietarios en cuya vivienda o local vivan, trabajen o presten servicios voluntarios, personas con discapacidad, o mayores de setenta años, con el objeto de asegurarles un uso adecuado a sus necesidades de los elementos comunes, así como la instalación de rampas, ascensores u otros dispositivos mecánicos y electrónicos que favorezcan la orientación o su comunicación con el exterior, siempre que el importe repercutido anualmente de las mismas, una vez descontadas las subvenciones 0 ayudas públicas, no exceda de doce mensualidades ordinarias de gastos comunes. No eliminará el carácter obligatorio de estas obras el hecho de que el resto de su coste, más allá de las citadas mensualidades, sea asumido por quienes las hayan requerido."

La Ley 8/2013- de Rehabilitación, Regeneración y Renovación Urbanas- LRRR, definen y obligan a realizar "Ajustes Razonables" de Accesibilidad en edificios existentes antes del 4 diciembre 2017, para asegurar la accesibilidad del parque edificado, dirigido a las viviendas colectivas y conocer la evaluación de las condiciones

WPS RI-SHUR, n4, 2016, vol.1, ISSN: 2387-1768

NÚMERO MONOGRÁFICO SOBRE GOBERNANZA URBANA/SPECIAL ISSUE ON GOVERNANCE 


\section{WPSReview International on Sustainable Housing and Urban Renewal}

(RI-SHUR)

básicas de Accesibilidad Universal. Se considera de interés señalar que en la parte II de ésta ley, se estudian las condiciones básicas de accesibilidad diferenciando entre uso residencial vivienda y uso residencial público y otros usos, comprobando de forma especial, si existen itinerarios accesibles en el exterior que permitan el acceso a todas las personas y sin discriminación, entre plantas o dentro de éstas en el mismo edificio.

En lo que corresponde a la Normativa específica de Accesibilidad en la Edificación, debemos señalar el Código Técnico de la Edificación -CTE y los Reglamentos Técnicos de Accesibilidad y Eliminación de Barreras de las distintas Comunidades Autonómicas, con diferentes grados restrictivos de aplicación de la accesibilidad.

La normativa sobre accesibilidad en el Código Técnico de la Edificación-CTE, está recogida en el Documento Básico de Seguridad, Usabilidad y Accesibilidad-DBSUA. En ocasiones por desconocimiento se dice que en el caso de adaptación de espacios, entornos y edificios a los parámetros de accesibilidad, solo es preciso aplicar el DBSUA9. Esto es un error, es preciso aplicar el DBSUA al completo, integrado por nueve documentos, siendo el DB-SUA9 el que hace referencia exclusiva a la accesibilidad.

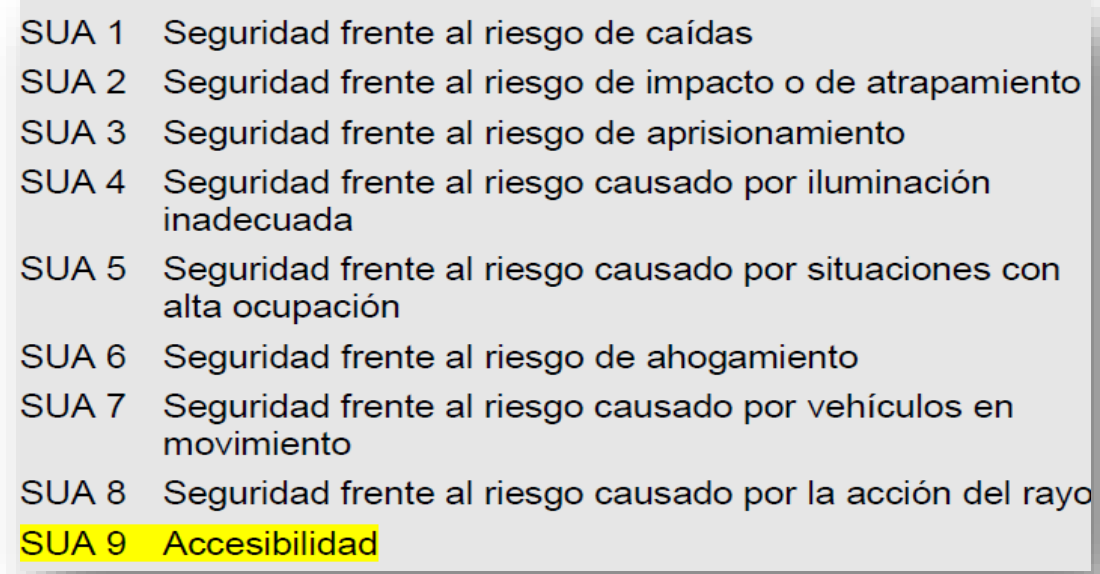

El objetivo del requisito básico "Seguridad de utilización y accesibilidad" es reducir a límites aceptables el riesgo de que los usuarios de un edificio sufran daños en el uso previsto del mismo de los edificios, como consecuencia de las características de su proyecto, construcción, uso y mantenimiento, así como en facilitar el acceso y la utilización no discriminatoria, independiente y segura de los mismos a las personas con discapacidad.

WPS RI-SHUR, n4ㄴ, 2016, vol.1, ISSN: 2387-1768

NÚMERO MONOGRÁFICO SOBRE GOBERNANZA URBANA/SPECIAL ISSUE ON GOVERNANCE 


\section{WPSReview International on Sustainable Housing and Urban Renewal}

(RI-SHUR)

Sin ninguna duda la dispersión de la normativa sobre Accesibilidad, dificulta su aplicación, ya que existe una Normativa de carácter Internacional ratificada por España, Normativa Estatal, Comunitaria y Local, debiendo conocer que siempre debemos aplicar la más restrictiva, como garantía que estamos asegurando la posibilidad de uso por personas con discapacidad. También debemos añadir que, aunque es preciso reconocer que se ha avanzado en los últimos años en la normativa sobre accesibilidad, es insuficiente. Si en las adaptaciones de edificios o entornos existentes aplicamos, exclusivamente, la normativa de obligado cumplimiento, es posible que los resultados obtenidos dificulten puedan ser utilizados por todos, por lo que es necesario aplicar recomendaciones de uso y buenas prácticas aplicadas, que garantizan la utilización por todos.

Por último, destacar el gran avance que supone la propuesta del "Acta Europea de Accesibilidad" (European Accessibility Act). Se trata de una Directiva Comunitaria, que supondrá un importante avance en el diseño de políticas que garanticen que todos los productos y servicios sean Accesibles para Todos.

Este Acta permitirá avanzar en la Inclusión Social de personas con discapacidad, en distintos aspectos esenciales que proporcionarán igualdad y la aplicación de los derechos escritos y reconocidos, pero no siempre aplicados. Se señalan los importantes avances que supondrá:

- Evitará la presencia de nuevas barreras en la ciudad y asegurará la igualdad de derecho para Todos los ciudadanos, sea cual sea sus capacidades.

- Obligará a los Estados miembros de la Convención de Naciones Unidas sobre Derechos las Personas con Discapacidad, entre otros España, a adoptar medidas para garantizar que las personas con discapacidad puedan acceder, en condiciones de igualdad, a los entornos, infraestructuras, transporte, información y comunicación, así como a cualquier servicio público.

- Unificará Criterios de Accesibilidad, fijando pautas comunes para todas las empresas. Tendrá gran repercusión en los derechos de las personas con discapacidad de la comunidad europea desarrollando acciones en áreas como las comunicaciones, servicios bancarios, telefonía, audiovisuales, comercio electrónico, transporte y ordenadores.

WPS RI-SHUR, n4, 2016, vol.1, ISSN: 2387-1768

NÚMERO MONOGRÁFICO SOBRE GOBERNANZA URBANA/SPECIAL ISSUE ON GOVERNANCE 


\section{4.- Resultados y Conclusiones}

\section{1.- Resultados}

Se han obtenido interesantes resultados en la gobernanza de las ciudades enfocados a los aspectos de inclusión de todas las personas, pero debemos añadir que el proceso es muy lento, lo que dificultad el uso de la ciudad por todas las personas. Por otra parte, la meta propuesta por ley para adaptación de todos los entornos y espacios públicos, es el 4 diciembre de 2017. Somos muchos los que apostamos que, considerando las fechas en las que nos encontramos y la falta de adaptación actual gran parte de los edificios y con carácter especial los de uso público, podemos concluir diciendo que es inviable. El Real Decreto Legislativo de 1/2013, que hemos citado con anterioridad, que aprueba el texto refundido de la Ley General de Derechos de las Personas con Discapacidad y de su Inclusión Social, reconoce la necesidad de adaptación de entornos y espacio para diciembre 2017 . Estudios realizados constatan que el $75 \%$ de los edificios, muestran una falta de adaptación, para su uso por personas con discapacidad, lo que cual exige tomar soluciones alternativas, ya la normativa va a afectar a la mayor parte del parque existente de edificios, por lo que se debería ir planificando las actuaciones necesarias y los costes correspondientes.

\section{2.- Conclusiones}

1. La Ciudad Inclusiva y Accesible es aquella que garantiza y considera los derechos de las personas con discapacidad y personas mayores, aplica la accesibilidad universal y diseño para todos en los distintos proyectos, la usabilidad de todos sus productos y servicios y diseña políticas de inclusión social para todos.

2. La Ciudad Inclusiva, respeta los criterios de Igualdad en la usabilidad de la ciudad, la empleabilidad, la educación, la sanidad y el uso de las tecnologías de información a disposición del ciudadano.

3. Los Gobiernos, las organizaciones gubernamentales, los profesionales y los ciudadanos son los responsables de crear y garantizar en su gobernanza la creación de Ciudades Inclusivas y Accesibles.

4. La gobernanza de una ciudad exige incorporar en sus políticas, proyectos y tecnologías de Accesibilidad para Todas las personas, en particular para aquellas con diferentes capacidades cognitivas, sensoriales, funcionales y personas mayores, incluyéndola como clave esencial de creación y adaptación de la ciudad.

WPS RI-SHUR, n4, 2016, vol.1, ISSN: 2387-1768

NÚMERO MONOGRÁFICO SOBRE GOBERNANZA URBANA/SPECIAL ISSUE ON GOVERNANCE 
5. Cada proyecto de una ciudad debe contemplar la accesibilidad desde el inicio y debe ser un parámetro esencial en todo el proceso de creación de la ciudad. Es un error la realización de capítulos o actuaciones independientes enfocadas a la mejora de la Accesibilidad de forma separada, excusa errónea para dar una imagen de accesible a los proyectos, pero nunca se obtiene la aplicación integral de la accesibilidad en el proyecto final.

6. La accesibilidad en las ciudades emergentes e inteligentes debe estar incluida en todos los "eslabones" que configuran la "Cadena de Accesibilidad", si se rompe en algunos de los eslabones, desaparece la accesibilidad en su conjunto.

7. Es preciso adaptar las infraestructuras existentes de las ciudades a los parámetros de Accesibilidad y Diseño para Todos, garantizando su uso por Todas las personas: Vivienda, Transporte, Educación, Cultura, puestos de trabajo, Sanidad y Turismo. El colectivo de personas con discapacidad integra uno de los grupos más marginados en el mundo, sin embargo, obtienen peores resultados sanitarios, obtienen peores resultados académicos, participan menos en la economía y disponen de menos empleabilidad.

8. En la gobernanza de las nuevas Ciudades o Ciudades Inteligentes la participación del ciudadano es fundamental, porque dinamiza y favorece su desarrollo. Del mismo modo se debe reconocer la presencia activa en la ciudad de las personas con diversidad funcional y discapacidad intelectual o del desarrollo, con la garantía que se asegura el desarrollo de su autonomía y usabilidad para este colectivo.

9. La propuesta de la Directiva Europea, con la inclusión del "Acta Europea de Accesibilidad", pendiente de su aprobación, supone un gran avance para la creación de Ciudades Innovadoras, Inteligentes Inclusivas y Accesibles.

10. La ciudad innovadora e inteligente debe presentarse como un documento en lectura fácil, un pictograma de servicios o un cartel orientativo. En definitiva, debe integrar a todas las personas y representar las distintas capacidades de las personas, sin olvidar de a las personas con discapacidad intelectual, lo cual obliga a aplicar "la accesibilidad cognitiva que también es un derecho".

WPS RI-SHUR, n4, 2016, vol.1, ISSN: 2387-1768

NÚMERO MONOGRÁFICO SOBRE GOBERNANZA URBANA/SPECIAL ISSUE ON GOVERNANCE 


\section{WPSReview International on Sustainable Housing and Urban Renewal}

(RI-SHUR)

\section{5.- Referencias Bibliográficas}

Allen, P.M. (1997) Cities and Regions as Self-Organizing Systems: Models of Complexity. Amsterdam: Gordon and Breach.

Caragliu, A., Del Bo, C. y Nijkamp, P. (2009). Smart cities in Europe. Vrije Universiteit.

Cerezuela Martínez, J.B.; Moreno Fernández, M.J.; y Sánchez Herrero, m.j. (2000): Los mayores y su hogar. Madrid: IMSERSO.

Couclelis, H. (2004). "The construction of the digital city". Planning and Design, 31(1), 519.

Dirección General de la Vivienda, la Arquitectura y el Urbanismo/Imserso (2002): Guía técnica de accesibilidad en la edificación" 2001. (2ª edición). Ministerio de Fomento.

Fernández Güell, Jose Miguel "Sostenibiidad gobernalidad: dos conceptos condenados a entenderse en el ámbito urbano", Madrid, mayo 2004

García Arias (2015), "Sistemas de indicadores de Smart Cities". XII Congreso Español de Ciencia Política y de la Administración. San Sebastian, 13-15 Jul.

Giffinger, R.; Fertner, C.; Kramar, H.; Kalasek, R.; Pichler-Milanovic, N. y Meijers, E. (2007). Smart Cities: Ranking of European Medium-Sized Cities. Viena: Centre of Regional Science (SRF) - Vienna University of Technology.

http://www.smart-cities.eu/download/smart_cities_final_report.pdf [Accedido el 19 de junio de 2015].

OCDE (2010) Green Cities Programme. http://www.oecd.org/gov/regional policy/49318965.pdf [Accedido el 19 de junio de 2015].

Parlamento Europeo (2014), Mapping Smart Cities in the EU [IP/A/ITRE/ST/2013- 02 January 2014 PE 507.480]. Bruselas: Unión Europea. http://www.smartcities.at/assets/Publikationen/Weitere-Publikationen-zum.

Thema/mappingsmartcities.pdf [Accedido el 19 de junio de 2015].

Willke, H. (2007). Smart governance: Governing the global knowledge society.

Chicago: University of Chicago Press.

Yovanof, G. S., y Hazapis G. N. (2009). "An architectural framework and enabling wireless technologies for digital cities and intelligent urban environments". Wireless Personal Communications, 49(3), 445-463.

WPS RI-SHUR, n4, 2016, vol.1, ISSN: 2387-1768

NÚMERO MONOGRÁFICO SOBRE GOBERNANZA URBANA/SPECIAL ISSUE ON GOVERNANCE 